\section{Interactive \\ ophthalmology: a smorgasbord of canine ophthalmology}

\section{David Maggs}

In this interactive session, we will work together through a series of cases using a problem-orientated approach that (while common in other branches of veterinary medicine) is often overlooked for ophthalmic diagnoses (Figure 1). However, this leads us to make only common diagnoses, and fail to consider alternative diagnoses when cases fail to improve. The problem-oriented approach progresses in a predictable manner from recognition of a problem (lesion, client complaint, altered clinical value), through mechanisms that cause that finding, to a clinical diagnosis. Application of the 'DAMNIT' list then ensures all etiological diagnosis are considered and guides diagnostic testing. This approach relies upon thorough history taking and a complete ophthalmic examination with identification of all (not just the major or the first-noted) abnormalities.

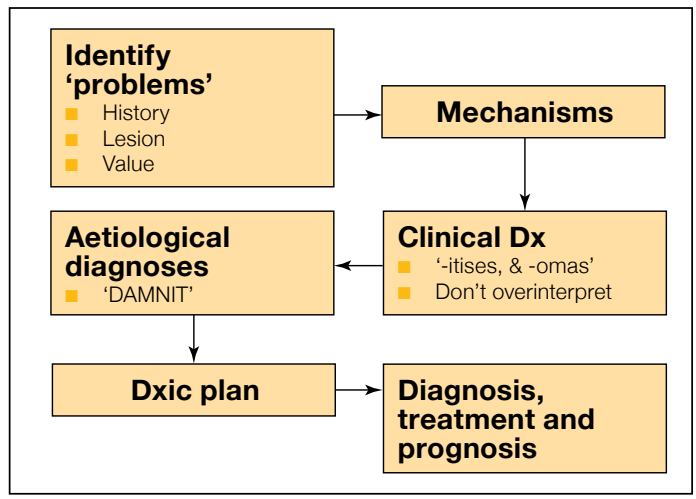

Figure 1: A problem-orientated approach to ophthalmology.

\section{STAGES IN THE DIAGNOSTIC PROCESS}

\section{Problems}

Our goal once we have completed taking a history and examining our patient is to come up with a succinct list of 'problems'. These might be historical complaints (e.g. sudden onset of blindness), abnormalities noted during our examination (e.g. aqueous flare) or altered measurements (e.g. low intraocular pressure). Therefore, problems are things that you detect and are described without interpretation. This avoids misinterpretation or 'jumping to conclusions'. For example, if we describe (actually, interpret) a reddened eye as having 'conjunctivitis' we will not have considered diseases such as uveitis, glaucoma, orbital disease, blepharitis, etc.

\section{Clinical diagnoses}

Clinical diagnoses can be thought of as '-acts, -itises and -omas' (e.g. blepharitis, conjunctivitis, cataract, glaucoma, etc.). Clinical diagnoses are interpretations of lesions but represent very general diagnoses since they can only be empirically treated. Before you initiate treatment, you should further define an aetiological diagnosis. For example, you may have noted ocular redness and clinically diagnosed it as conjunctivitis, but knowing the cause of that conjunctivitis is essential before prescribing a treatment. Consider whether you would use the same treatment for foreign body conjunctivitis in a dog and herpesviral conjunctivitis in a cat. That's where the DAMNIT list comes in.

\section{Aetiological diagnoses}

The DAMNIT list used in other specialities applies equally well to ophthalmic conditions. For instance cataracts may be Degenerative (e.g. senile), Developmental or Anomalous (e.g congenital), Metabolic (e.g. diabetes mellitus), Nutritional (e.g. arginine deficiency), Inflammatory (e.g. secondary to uveitis), Infectious (e.g. Diplostomum spathaceum - a trematode in fish!), Traumatic, or Toxic (e.g. heavy metal poisoning). All possible causes should be considered for each clinical diagnosis, but when an inflammatory clinical diagnosis (i.e. an '-itis') is made, initial emphasis can be placed upon infectious, immune-mediated, neoplastic and traumatic aetiologies. The list of potential aetiological ('differential') diagnoses produced by the DAMNIT approach is then tested (intellectually and using diagnostic tests) for likelihood, and ranked. Using 'response to therapy' as a diagnostic test is sometimes reasonable, especially if this problem-based approach has been taken and if it has been verified that the proposed treatment trial is unlikely to worsen one of the other (presumably less likely) differential diagnoses.

\section{KEY LEARNING OBJECTIVES}

- To differentiate and apply 'lesions', 'clinical diagnoses', and 'aetiological diagnoses' as they apply to a problem-based approach to ocular disease

- To apply the DAMNIT system to ocular diagnoses

- To apply a problem-based approach to ocular diseases seen commonly in your practice

\section{MULTIPLE CHOICE QUESTIONS}

1. What is conjunctivitis?
a. Lesion or problem
b. Clinical diagnosis
c. Aetiological diagnosis
d. None of the above

2. What does applying the DAMNIT scheme permit?
a. Identification of problems
b. Making of clinical diagnoses
c. Selection of appropriate therapy
d. Some diagnostic tests to be omitted

3. What is the problem-orientated approach designed to do?
a. Ensure lesions are not overlooked
b. Establish a systematic means of approaching ocular diagnoses
c. Improve patient outcomes 
d. All of the above

4. Which of the following about uveitis is correct?
a. It is an aetiological diagnosis
b. It can be treated only empirically until a cause is identified
c. It is a lesion exemplified by low intraocular pressure
d. Does not require a problem-orientated approach

5. What does the problem-based approach necessitate?
a. Identifying all lesions that exist
b. Finding sufficient clinical diagnoses to explain all lesions
c. Applying the DAMNIT list to identify aetiological diagnoses
d. All of the above 